\title{
Molecular genetics of B-precursor acute lymphoblastic leukemia
}

\author{
Charles G. Mullighan \\ Department of Pathology, St. Jude Children's Research Hospital, Memphis, Tennessee, USA.
}

\begin{abstract}
B-precursor acute lymphoblastic leukemia (B-ALL) is the most common childhood tumor and the leading cause of cancer-related death in children and young adults. The majority of B-ALL cases are aneuploid or harbor recurring structural chromosomal rearrangements that are important initiating events in leukemogenesis but are insufficient to explain the biology and heterogeneity of disease. Recent studies have used microarrays and sequencing to comprehensively identify all somatic genetic alterations in acute lymphoblastic leukemia (ALL). These studies have identified cryptic or submicroscopic genetic alterations that define new ALL subtypes, cooperate with known chromosomal rearrangements, and influence prognosis. This article reviews these advances, discusses results from ongoing second-generation sequencing studies of ALL, and highlights challenges and opportunities for future genetic profiling approaches.
\end{abstract}

\section{Introduction}

Acute lymphoblastic leukemia (ALL) is a neoplasm of immature lymphoid progenitors that is most commonly of B cell lineage. Most childhood cases of B-precursor ALL (B-ALL) may be subclassified by the presence of either gross or submicroscopic genetic alteration. Approximately $75 \%$ of B-ALL cases exhibit aneuploidy or a recurring gross chromosomal rearrangement $(1,2)$ (Table 1 and Figure 1). Hyperdiploidy, with gain of at least 5 chromosomes, is one of the most frequent alterations in childhood ALL and is associated with favorable outcome (3). However, the biologic basis of the acquisition of multiple whole chromosomal gains is poorly understood. Conversely, hypodiploidy, with fewer than 44 chromosomes, is associated with dismal prognosis (4). A number of recurring chromosomal rearrangements are common in B-ALL and are critical events in leukemogenesis. These rearrangements commonly perturb genes encoding regulators of hematopoiesis, tumor suppressors, oncogenes, or tyrosine kinases but commonly require additional genetic hits to establish the full leukemic phenotype.

In the last five years, genome-wide profiling using microarrays, candidate gene, and second-generation sequencing have provided a number of key insights into the genetic basis of ALL. These studies have identified new subtypes of ALL and have uncovered recurring submicroscopic genetic alterations in known ALL subtypes. These include loss-of-function mutations involving genes regulating lymphoid development that contribute to the arrest in maturation characteristic of B-ALL, mutations that inactivate tumor suppressor and cell cycle regulatory proteins, and mutations that drive cytokine receptor and/or kinase signaling. Thus, as in AML, concomitant lesions disrupting hematopoietic development and tumor suppression as well as driving signaling and proliferation are hallmarks of many ALL subtypes. Importantly, several of these alterations are associated with specific subtypes of ALL defined by recurring chromosomal alterations.

This Review describes the genetic landscape of ALL, focusing on important subtypes and recently discovered genetic alterations of biologic and therapeutic relevance.

Conflict of interest: The author has declared that no conflict of interest exists. Citation for this article: J Clin Invest. 2012;122(10):3407-3415. doi:10.1172/JCI61203.

\section{ETV6-RUNX1 (TEL-AML1) B-ALL}

The most common rearrangement in B-ALL is the $t(12 ; 21)$ (p13;q22) rearrangement that encodes ETV6-RUNX1 (5). This rearrangement is usually cryptic on cytogenetic analysis but is readily detected by fluorescent in situ hybridization and molecular techniques. ETV6 is a member of the ETS family of transcription factors that are frequently targeted by rearrangements and mutations in leukemia and other malignancies (6). With CBFB, RUNX1 forms the core binding factor transcription complex and is commonly rearranged in acute myeloid leukemia (7), and harbors sequence mutations in myeloid and lymphoid disorders (8-10). Both ETV6 and RUNX1 are required for normal definitive hematopoiesis $(7,11)$, and the ETV6-RUNX1 protein may perturb expression of RUNX1-regulated genes, converting RUNX1 to a transcriptional repressor (12). ETV6-RUNX1 also causes overexpression of the erythropoietin receptor (EPOR) and activation of downstream JAK-STAT signaling (13). Expression of ETV6-RUNX1 promotes self-renewal in B cell progenitors but alone does not induce leukemia $(14,15)$. Furthermore, ETV6-RUNX1 is commonly detectable at birth (16), years prior to the onset of leukemia, suggesting that secondary genetic events are required to induce leukemia. This model is supported by genome-wide profiling studies of genetic alterations in ALL that have identified additional recurring submicroscopic genetic alterations in ETV6-RUNX1 ALL, including deletions of the B cell transcription factors $P A X 5$ and $E B F 1$ and deletion of the second copy of ETV6 (17-19). Moreover, monozygotic twins concordant for ETV6-RUNX1 ALL exhibit distinct submicroscopic DNA copy number alterations (CNAs), indicating that acquisition of the ETV6-RUNX1 is an early event in leukemogenesis (20). Submicroscopic genetic alterations are more common in ETV6-RUNX1 ALL than in many other ALL subtypes and include deletions targeting putative lymphoid signaling molecules (BTLA, TOX), transcriptional coactivators (TBL1XR1), the glucocorticoid receptor gene NR3C1, and the putative apoptosis regulatory gene BTG1. Although these alterations may directly contribute to leukemogenesis, the genomic breakpoints of many of these deletions bear the hallmark of aberrant activity of the recombinase activation genes. Thus, further work directly examining these alterations in leukemogenesis is required. 


\section{Table 1}

Key cytogenetic alterations and genetic subtypes in childhood B-ALL

\begin{tabular}{|c|c|c|c|}
\hline Subtype & Frequency (\%) & Comment & References \\
\hline Hyperdiploidy (>50 chromosomes) & $20-30$ & Excellent prognosis with antimetabolite-based therapy & 3 \\
\hline Hypodiploidy (<44 chromosomes) & $1-2$ & $\begin{array}{l}\text { Poor prognosis, high frequency of RAS pathway and } \\
\text { IKAROS gene family mutations }\end{array}$ & 4 \\
\hline $\mathrm{t}(12 ; 21)(\mathrm{p} 13 ; \mathrm{q} 22)$ ETV6-RUNX1 & $15-25$ & Expression of myeloid antigens; excellent outcome & 5 \\
\hline $\mathrm{t}(1 ; 19)(\mathrm{q} 23 ; \mathrm{p} 13)$ TCF3-PBX1 & $2-6$ & $\begin{array}{l}\text { Increased incidence in African Americans; } \\
\text { generally excellent prognosis; association with CNS relapse }\end{array}$ & $121-123$ \\
\hline $\mathrm{t}(9 ; 22)(\mathrm{q} 34 ; \mathrm{q} 11.2) B C R-A B L 1$ & $2-4$ & $\begin{array}{l}\text { Historically dismal outcome, improved with addition } \\
\text { of imatinib to intensive chemotherapy }\end{array}$ & 39,42 \\
\hline$P A X 5$ rearrangement & $\sim 2 \%$ & $\begin{array}{l}\text { Multiple partners, commonly from } \operatorname{dic}(7 ; 9) \text {, dic/t(9;12) } \\
\text { and dic }(9 ; 20) ; \text { outcome unknown }\end{array}$ & 17,94 \\
\hline$A B L 1, P D G F R B, J A K 2$ rearrangements & $2-5$ & $\begin{array}{l}\text { Identified in BCR-ABL1-like ALL; multiple rearrangements encoding } \\
\text { chimeric proteins, fusing 5' partners with } 3^{\prime} \text { kinase domains; } \\
\text { associated with IKZF1 alteration and very high leukocyte count; } \\
\text { potentially amenable to tyrosine kinase inhibitor therapy }\end{array}$ & 84 \\
\hline $\mathrm{t}(4 ; 11)(\mathrm{q} 21 ; \mathrm{q} 23) M L L-A F 4$ & $1-2$ & $\begin{array}{l}\text { Common in infant ALL (especially }<6 \text { months of age); } \\
\text { poor prognosis }\end{array}$ & 45 \\
\hline $\begin{array}{l}\text { MYC rearrangement }[\mathrm{t}(8 ; 14)(\mathrm{q} 24 ; q 32) \text {, } \\
\mathrm{t}(2 ; 8)(\mathrm{q} 12 ; \mathrm{q} 24)]\end{array}$ & 2 & Favorable prognosis with short-term, high-dose chemotherapy & 70 \\
\hline $\begin{array}{l}\text { CRLF2 rearrangement (IGH@-CRLF2, } \\
\text { PAR1 deletion, and P2RY8-CRLF2) }\end{array}$ & $5-7$ & $\begin{array}{l}\text { Extremely common in DS ALL }(55 \%) \text {; association with } \\
\text { IKZF1 deletion/mutation and JAK1/2 mutation; } \\
\text { poor prognosis in non-DS ALL }\end{array}$ & $71-75$ \\
\hline ERG deletion & 7 & $\begin{array}{l}\text { Subtype of B-ALL with a distinct gene expression profile; } \\
\text { favorable outcome }\end{array}$ & 124 \\
\hline
\end{tabular}

\section{TCF3-PBX1 (E2A-PBX1) and TCF3-HLF (E2A-HLF) B-ALL}

The $t(1 ; 19)(\mathrm{q} 23 ; \mathrm{p} 13)$ translocation is present in up to $6 \%$ of childhood B-ALL cases (21). The rearrangement is commonly unbalanced, with duplication of $1 \mathrm{q}$ distal to $P B X 1$.

This translocation fuses the transactivation domains of TCF3 with the C-terminal region of the homeobox gene PBX1. TCF3 encodes the basic helix-loop-helix (bHLH) transcription factors E12 and E47 that are required for early lymphoid development, and loss of E12/E47 promotes the development of $\mathrm{T}$ cell lymphoma $(22,23) . P B X 1$, which encodes a member of the three-amino acid loop extension family of homeodomain proteins, is required for the development of lymphoid precursors (24). TCF3-PBX1 binds HOX proteins and likely interferes with hematopoietic differentiation by disrupting HOX-regulated gene expression (25).

The $t(17 ; 19)(q 22 ; p 13)$ translocation is a rare rearrangement that fuses the aminoterminal transactivation domains of TCF3 to the C-terminal DNA-binding and dimerization domains of HLF, a member of the PAR family of basic leucine zipper transcription factors (26). TCF3-HLF aberrantly regulates genes that control cell death in lymphoid progenitors, including LMO2 and BCL2 (27). TCF3-HLF-positive leukemia is associated with older age and very poor outcome (28).

\section{BCR-ABL1 ALL}

Breakpoint cluster region-ABL1 (BCR-ABL1) is generated by the der(22) of the $\mathrm{t}(9 ; 22)$ (q34;q11) translocation, or Philadelphia (Ph) chromosome, which is present in over $95 \%$ of chronic myelogenous leukemia (CML) cases, $25 \%$ of adult ALL cases, and $3 \%$ to $5 \%$ of pediatric ALL cases (29). The $t(9 ; 22)$ fuses 5 ' sequences of breakpoint cluster region $(B C R)$ to $3^{\prime}$ sequences from $A B L 1$, which encodes a tyrosine kinase. The breakpoints on chromosome 9 are scattered over a nearly 200-kb region within the first intron of $A B L$, whereas the BCR breakpoints on chromosome 22 are clustered in two areas: a 5.8-kb major BCR (M-bcr) in CML and a minor BCR (m-bcr) in most cases of childhood Ph-positive ALL (30). Fusion genes created by breaks in M-bcr (CML-type break) encode a 210-kDa fusion protein ( 210$)$, whereas fusions that occur in m-bcr (ALL-type break) encode $\mathrm{p} 190$.

Both forms of BCR-ABL1 can transform hematopoietic cells in vitro and induce a syndrome similar to CML in mice (31). BCRABL1 activates multiple signaling pathways, increases cell proliferation, and deregulates differentiation and adhesion. Treatment of BCR-ABL1-positive leukemic cells with tyrosine kinase inhibitors results in the activation of the transcription factor BCL6, which may directly influence responsiveness to treatment with these agents (32).

Additional genetic alterations are critical determinants of the lineage and progression of BCR-ABL1 leukemia. Deletion of the early lymphoid transcription factor gene IKZF1 (IKAROS) is common in BCR-ABL1 lymphoid leukemia (CML at progression to lymphoid blast crisis) but is rarely present in CML at chronic phase $(33,34)$. IKAROS is a zinc finger transcription factor required for the development of all lymphoid lineages $(35,36)$. The IKZF1 deletions result either in haploinsufficiency or expression of dominant-negative isoforms. These alterations cooperate with $B C R-A B L 1$ in the induction of lymphoblastic leukemia and promote resistance to therapy in recent experimental models of BCR-ABL1 ALL (37).

In childhood ALL, the presence of BCR-ABL1 is associated with older age, higher leukocyte count, and more frequent CNS leukemia at diagnosis (38). Historically, BCR-ABL1 ALL has been associated with extremely poor outcome $(29,39)$. Tyrosine kinase inhibitors 


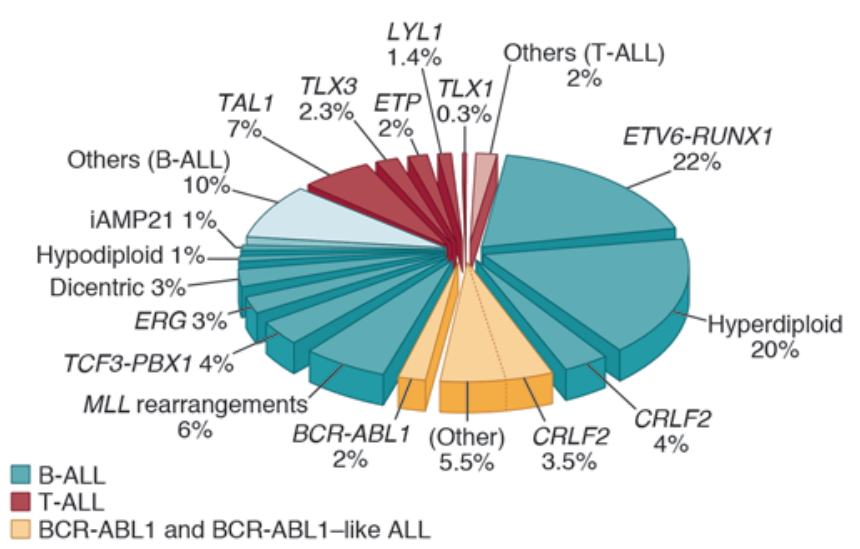

such as imatinib mesylate (Gleevec) have transformed the treatment and outcome of patients with BCR-ABL1-positive leukemia $(40,41)$. The addition of imatinib to intensive chemotherapy in childhood BCR-ABL1-positive ALL results in a 4-year event-free survival rate of $84 \%$, more than double that of historical controls (42). A proportion of patients become resistant to these agents, typically through acquired mutations in the ABL1 kinase domain (43).

\section{MLL-rearranged B-ALL}

Mixed-lineage leukemia-rearranged ( $M L L$-rearranged) leukemia is a unique entity notable for initiation in utero, for myeloid and lymphoid features, and for poor responsiveness to therapy (44, 45). Rearrangement of the $M L L$ gene at $11 \mathrm{q} 23$ occurs in at least two-thirds of infants with ALL, $5 \%$ of AML, and $85 \%$ of secondary leukemias that occur in patients treated with topoisomerase II inhibitors $(46,47)$ but is less frequent in older individuals $(48,49)$. Over 80 partners of $M L L$ rearrangement have been identified (50).

The most common $M L L$ rearrangements are $\mathrm{t}(4 ; 11)(\mathrm{q} 21 ; \mathrm{q} 23) /$ $M L L-A F F 1(A F 4)$, found in approximately $50 \%$ of cases, followed by $\mathrm{t}(9 ; 11)(\mathrm{p} 22 ; \mathrm{q} 23) / M L L-M L L T 3(A F 9), M L L-E N L$, and $\mathrm{t}(10 ; 11)$ (p13-15;q14-21)/MLL-MLLT10(AF10) $(51,52)$. The translocation breakpoints are located in an 8.5 -kb cluster between exons 5 and 11 and juxtapose the A-T hook and methyltransferase domains to partner proteins. MLL regulates hematopoiesis through maintenance of normal homeotic gene expression (53), in part through transcriptional activation of HOX genes, mediated though the histone $\mathrm{H} 3$ lysine 4 (H3K4) methyltransferase activity of the SET domain (54). In contrast to other subtypes of B-ALL, additional genetic alterations are uncommon in $M L L$ rearranged leukemia $(17,55,56)$.

$M L L$-rearranged ALL exhibits a distinct gene expression profile characterized by the upregulation of class I HOX genes (HOXA5, $-A 7$, and - $A 9$ and the HOX cofactor gene MEIS1) (57). Co-expression of HOXA genes and MEIS1 cooperates with MLL fusions in induction of leukemia and maintenance of a "stem cell-like" state of maturation (58). fms-related tyrosine kinase 3 (FLT3) is overexpressed in MLL-rearranged ALL and harbors point mutations in $10 \%-20 \%$ of cases (59). In animal models, FLT3 kinase inhibitors have shown potential as therapeutic agents for $M L L$ rearranged leukemias, and FLT3 inhibitors are currently being tested in clinical trials $(59,60)$. $M L L$-rearranged leukemias also exhibit a distinct epigenetic profile, with signatures of cytosine (61), microRNA (62) and H3K79 methylation (63) that differ from non- $M L L$ leukemias.

\section{Figure 1}

Frequency of cytogenetic subtypes of pediatric ALL. The pie chart includes all major B-ALLs and T-lineage subtypes of ALL, to illustrate the relative frequency of each. The recently described BCR-ABL1-like subtype and BCR-ABL1-positive ALL are shown in yellow to illustrate the high frequency of childhood B-ALL cases with genetic alterations activating tyrosine kinase and cytokine receptor signaling that may be amenable to targeted therapy. Data are derived from front-line studies of childhood ALL (121).

Many of the MLL fusion proteins are located in protein complexes that regulate transcriptional elongation, which may be in part responsible for transcriptional dysregulation and leukemogenesis. These include polymerase-associated factor complex, which associates with the N terminus of MLL and regulates RNA polymerase II (Pol II), the pTEFb (CDK9/cyclin T) complex, which associates with the fusion partners MLLT1 (ENL), ELL, and AFF1 (AF4) to release stalled Pol II and stimulate transcriptional elongation, and the H3K79 histone methyltransferase DOT1L, which binds MLL fusion partners including AFF1, AF9, AF10, and ENL (64). H3K79 methylation is a mark of actively transcribed genes, and suppression or inactivation of DOT1L in human or murine leukemia cells leads to suppression of the MLL fusion proteininduced gene expression program (63), differentiation and/or apoptosis of leukemic cell lines, and suppression of leukemogenesis (65). DOT1L inhibition is thus being pursued as a therapeutic approach in $M L L$-rearranged ALL (66).

These findings highlight the potential importance of epigenetic dysregulation in other subtypes of ALL. Several studies profiling cytosine methylation have shown that different ALL subgroups exhibit distinct methylation profiles and that promoter methylation is associated with gene expression and outcome $(67,68)$. Additional studies are needed to comprehensively determine the relative importance of genetic and epigenetic alteration in the pathogenesis of ALL as well as the potential therapeutic role of epigenetic modifying drugs.

\section{Rearrangement of CRLF2 and other immunoglobulin heavy locus rearrangements in B-ALL}

Rearrangements of the immunoglobulin heavy locus (IGH@) at 14q32.33, resulting in juxtaposition of IGH@ enhancer elements with transcription factor and cytokine receptor genes, are observed in both B-ALL and T-lineage ALL (69). B-lineage leukemia/lymphoma with rearrangement of MYC is reviewed elsewhere (70).

Cytokine receptor-like factor 2 (CRLF2; also known as thymic stromal-derived lymphopoietin [TSLP] receptor) is located at the pseudoautosomal region (PAR1) at Xp22.3/Yp11.3 and is rearranged or mutated in up to $7 \%$ of B-ALL cases $(71,72)$. With the IL-7R $\alpha$ chain, CRLF2 forms a heterodimeric receptor for TSLP. The rearrangements are either rearrangement into IGH@-CRLF2 or focal deletion immediately upstream of CRLF2, resulting in a chimeric fusion of the purinergic receptor gene $P 2 R Y 8$ to the entire coding region of CRLF2. Both rearrangements result in overexpression of CRLF2 on the cell surface (71). Less commonly, 
a p.Phe232Cys mutation in the transmembrane domain of CRLF2 results in receptor dimerization and overexpression (73).

CRLF2 rearrangements are present in at least $50 \%$ of Down syndrome-associated (DS-associated) B-ALL cases $(71,72)$ and are also present in up to $50 \%$ of a novel subtype of high-risk B-ALL, BCR-ABL1-like ALL (see below) (74). CRLF2 alterations are associated with the presence of activating mutations in the Janus kinase genes JAK1 and JAK2 $(71-73,75)$, which are otherwise uncommon in B-ALL. The JAK mutations in B-ALL most commonly disrupt p.Arg683 in the pseudokinase domain of JAK2, in contrast to the JAK2 p.Val617Phe mutations that are a hallmark of myeloproliferative diseases. Like the JAK2 p.Val617Phe mutation, the JAK1/2 mutant alleles observed in ALL confer cytokine-independent proliferation in cultured cells, particularly in the presence of a cytokine receptor such as the EPOR or CRLF2, suggesting that a receptor scaffold is required for mutant JAK alleles to drive proliferation and lymphoid transformation $(76,77)$. In non-DS ALL, CRLF2 rearrangements are associated with IKZF1 alteration, JAK mutation, and poor outcome $(74,78)$. Recently, activating mutations in IL-7R have been identified in both B-ALL and T-lineage ALL, including cases of CRLF2 rearrangement $(10,79,80)$. Thus, the use of JAK inhibitors is actively being pursued to target leukemic cells harboring CRLF2/JAK alterations and leukemic cells with IL-7R mutations that also activate JAK/STAT signaling. Several otherIGH@ translocation partners have been identified in B-ALL, including EPOR, the CEBP transcription factor gene family, the inhibitor of DNA binding 4 (ID4) gene, the LIM domaincontaining protein LHX4, and rarely, IL-3 (69).

\section{BCR-ABL1-like ALL}

Up to $15 \%$ of childhood B-ALL cases exhibit a gene expression profile similar to that of BCR-ABL1-positive ALL, often have deletion/mutation of IZKF1, and have a very poor outcome (81-83). CRLF2 is rearranged in up to $50 \%$ of BCR-ABL1-like ALL cases, but until recently the lesions responsible for kinase activation in the remaining cases were unknown. Second-generation mRNA sequencing and whole genome sequencing of 15 BCR-ABL1-like ALL cases identified rearrangements, sequence mutations, and DNA CNAs activating kinase signaling in all cases (84), including rearrangements of PDGFRB, ABL1, JAK2, and EPOR as well as deletion/mutation of SH2B3 and IL7R. Several of these alterations have been shown to result in activation of downstream (e.g., JAK/STAT) signaling pathways, and cellular transformation was attenuated with JAK or ABL1/PDGFRB inhibitors. Thus, defining kinase-activating alterations in this subtype of ALL is critical to identifying and treating patients with this subtype of leukemia.

\section{Intrachromosomal amplification of chromosome $\mathbf{2 1}$}

Intrachromosomal amplification of chromosome 21 (iAMP21) occurs in up to $2 \%$ of B-progenitor ALL cases (85). The region of amplification is typically large but variable, but includes gain of at least three copies of RUNX1, commonly with deletion of the subtelomeric regions of chromosome 21. iAMP21 is characterized by commonly complex patterns of chromosomal rearrangement suggestive of a breakage-fusion-bridge cycle (86). iAMP21 frequently occurs in older children or adolescents and has been reported to be associated with poor outcome (87), although this association may not be independent of other established prognostic variables such as levels of minimal residual disease (88).

\section{Hypodiploid ALL}

Hypodiploidy, with fewer than 44 chromosomes, is present in up to $3 \%$ of childhood ALL cases and is associated with a high risk of treatment failure (89). Hypodiploid ALL may be subclassified by severity of aneuploidy into near-haploid (NH; 24-31 chromosomes) and low-hypodiploid ( $\mathrm{LH} ; 32-44$ chromosomes) cases, but until recently the concomitant submicroscopic genetic alterations were poorly characterized. NH ALL has a very high frequency of deletions and sequence mutations that activate RAS signaling, and $\mathrm{NH}$ and LH ALL have a high frequency of inactivating alterations of IKAROS genes IKZF2 (HELIOS) and IKZF3 (AIOLOS) that are otherwise rare in ALL (90). Moreover, LH cases have a very high frequency of loss-of-function mutations in TP53 (encoding p53) and RB1, which are otherwise uncommon at diagnosis in B-ALL.

\section{Submicroscopic genetic alterations in B-ALL}

The observation that up to $25 \%$ of children with ALL lack a recurring chromosomal alteration (2) and the identification of translocation-encoded fusions at birth or in years prior to the onset of leukemia (16) indicates that additional genetic alterations cooperate in leukemogenesis. The advent of array-comparative genomic hybridization and SNP microarrays has enabled interrogation of structural genetic alterations at very high resolution (91). These approaches, coupled with candidate gene sequencing and integrated gene expression profiling (92), have identified a number of critical new targets of mutation in B-ALL that often involve genes that regulate lymphoid development, cell cycle, tumor suppression, and a variety of other key cellular pathways (Table 2). The mean number of DNA CNAs is lower than in many solid tumors (an average of 6-8 lesions per case) $(17,19,33)$, but over 50 recurring CNAs have been identified. The nature and frequency of these CNAs are significantly associated with ALL subtype, with few lesions in MLL-rearranged ALL $(17,55)$ but 6-8 lesions per case in BCR-ABL1 and ETV6-RUNX1 ALL $(33,34)$.

Most notably, genes encoding transcriptional regulators of B-lineage commitment and differentiation are mutated in over two-thirds of B-ALL. These include PAX5 (encoding paired box 5, mutated in over $30 \%$ of cases), EBF1 (early B cell factor 1), IKZF1-3, and lymphoid enhancer factor 1. PAX5 harbors broad deletions predicted to result in haploinsufficiency, focal internal deletions that result in the expression of truncated PAX5 transcripts, sequence mutations that disrupt DNA binding or truncate the transactivating domain $(17,93)$, and translocations that create chimeric fusion proteins $(17,93,94)$. These lesions usually only involve a single copy of PAX5, and expression of key PAX5 targets, such as CD19, in ALL cells is frequently normal, suggesting that PAX5 haploinsufficiency contributes to leukemogenesis.

Despite their high frequency of occurrence, $P A X 5$ alterations are not associated with outcome in ALL $(81,95)$. In contrast, IKZF1 alterations are less frequent (15\% of B-ALLs) but are associated with adverse outcome in both BCR-ABL1-positive and negative ALL cases. IKZF1 alterations are present in up to one-third of highrisk B-ALL cases and triple the risk of treatment failure $(81,96)$. IKZF1, which is required for lymphoid development, binds DNA and forms homodimers or heterodimers with other IKAROS family members. IKZF1 has multiple functions including transcriptional activation, repression, and chromatin remodeling, in part mediated via the nucleosome remodeling and deacetylase complex that also contains IKZF3. The alterations observed in ALL include broad and focal deletions of IKZF1, which result in loss of func- 
review series

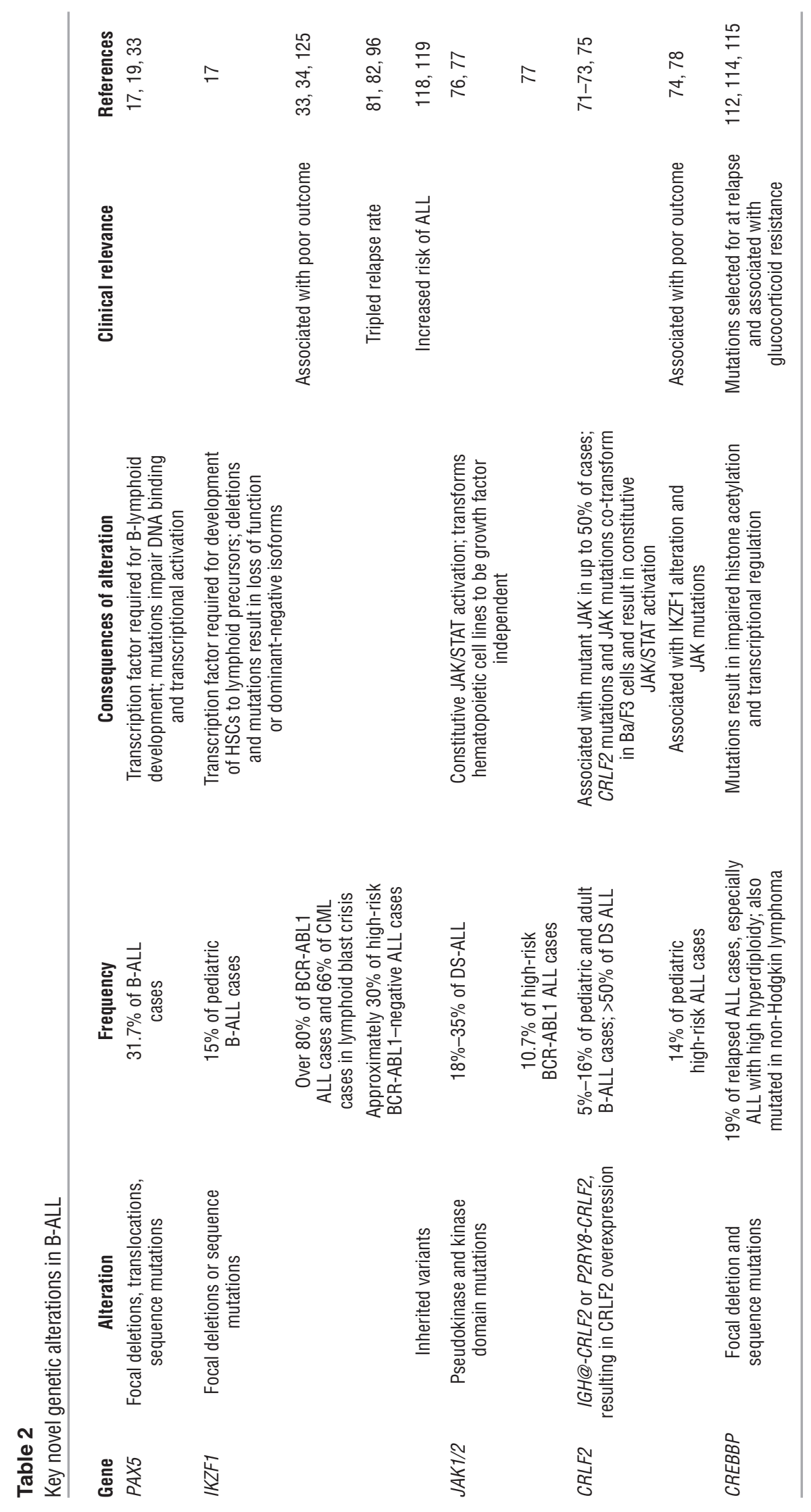




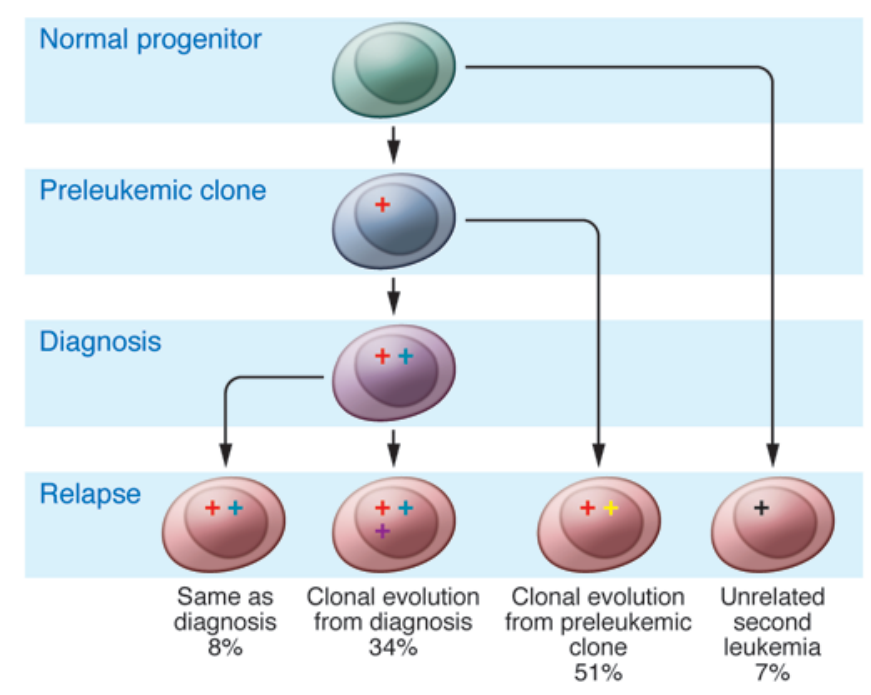

tion, focal internal deletions of exons 4-7, resulting in expression of the dominant-negative isoform IK6, and less commonly, loss-offunction and dominant-negative sequence mutations. How these IKZF1 alterations contribute to leukemogenesis remains poorly understood. IKZF1 inactivation likely contributes to the arrest in maturation characteristic of ALL, and loss of Ikzfl, like that of Pax5, accelerates BCR-ABL1 lymphoid leukemia development in murine models (37). However, the striking association of IKZF1 alterations with poor outcome suggests that other mechanisms may be involved. Detection of IKZF1 alterations at diagnosis to aid risk stratification and treatment assignment is consequently under investigation in ongoing prospective clinical trials.

Additional recurring genetic alterations in B-ALL target cell cycle regulation, transcriptional activation, tumor suppression, lymphoid signaling, apoptosis, and drug responsiveness. These include CDKN2A/CDKN2B, RB1, CD200, BTLA/TOX, BTG1, the glucocorticoid receptor NR3C1, TBL1XR1, ETV6, and ERG (17-19, 97). Candidate gene sequencing approaches have identified additional sequence mutations in B-ALL, most commonly mutations resulting in activation in RAS signaling, which are enriched in $M L L$ and hyperdiploid ALL $(98,99)$. Additional pathways targeted include JAK/STAT signaling, the TP53/RB1 pathway, and lymphoid development and additional mutations have been found in transcriptional coactivators, including ERG, TBL1XR1, CTCF, $C R E B B P$, and EP300 (100).

\section{Genetics of relapse in ALL}

ALL genomes are not static, frequently acquiring secondary karyotypic and genetic alterations during disease progression (101-103). Genome-wide profiling has enabled key questions in the genetic basis of treatment failure to be investigated, including the nature of genetic alterations that are acquired with disease progression, the genetic relationship between the diagnosis and relapsed leukemic clones, and the degree of clonal heterogeneity present at the time of diagnosis.

Microarray profiling has shown that relapse samples exhibit striking differences in the patterns of genomic alterations from the matched diagnosis samples (104-108), with the majority of relapse samples exhibiting the loss of CNAs present at diagnosis and the acquisition of new genetic alterations. Importantly, most

\section{Figure 2}

Patterns of genomic evolution from diagnosis to relapse in ALL. Leukemic clones at relapse are frequently related to leukemic clones present at diagnosis. In more than half of cases, the relapse clone arises from a clone present prior to diagnosis, retaining some but not all of the lesions found at diagnosis and containing some additional mutations. The relapse clone is often present as a rare subclone within the diagnostic sample. Alternatively, cases of relapse can stem from the diagnosis clone, acquiring additional mutations. Less commonly, the relapse clone may be identical to the diagnosis clone or appear to be an unrelated second leukemia.

paired diagnosis-relapse samples share a common clonal origin. Moreover, lesions detected at relapse are often detectable in diagnostic samples, indicating that the predominant relapse clone(s) are present at low levels at diagnosis. Genetically unrelated second leukemias (at least at the level of DNA CNA analysis by SNP array) are infrequent but may be more common in late relapse (109). Approximately one-third of cases show a pattern of linear clonal evolution (with the acquisition of new genetic changes by the relapse clone in addition to those seen at relapse), and over $50 \%$ show a complex picture with both loss of diagnosis CNAs and the acquisition of new lesions, as well as evidence of low levels of relapse clones at diagnosis (Figure 2).

Together, these data suggest that a pre-diagnosis "ancestral" clone harboring one or more genetic alterations (such as a founding translocation) undergoes divergent evolution into multiple clones that acquire different genetic alterations and emerge as the predominant clones at diagnosis and relapse. This is supported by studies of monozygotic twins concordant for ALL, showing different secondary CNAs in each twin (20), and recent xenotransplantation studies that have modeled clonal evolution and heterogeneity by engrafting ETV6-RUNX1 and BCR-ABL1 samples into immunocompromised mice. These studies have confirmed clonal heterogeneity in the majority of cases at the time of diagnosis and have shown that specific genetic alterations (e.g., deletion of CDKN2A/CDKN2B) influence the efficiency and tempo of engraftment $(110,111)$.

Genome-wide sequencing is required to comprehensively identify all sequence and structural variants contributing to the pathogenesis of ALL, and a number of sequencing efforts are ongoing. However, a recent study performed Sanger sequencing of 300 genes in 23 matched diagnosis-relapse samples, with recurrence testing of over 300 paired and unpaired ALL samples (112). This study identified a number of uncharacterized mutations in ALL, most notably deleterious mutations in CREBBP (encoding CREBbinding protein) in over $20 \%$ of relapse ALL samples (112). The mutations were present at relapse and were detected in either the predominant clone or a subclone at diagnosis. CREBBP is a transcriptional coactivator, histone and non-histone acetylase, and ubiquitin ligase (113). Mutations were common in the histone acetyl transferase (HAT) domain and resulted in impaired acetylation of histone targets (112). CREBBP mutations are highly enriched 
in hyperdiploid ALL (114) and are common at diagnosis in B cell lymphoma samples (115). Importantly, the HAT mutations impair the normal CREBBP-mediated transcriptional response to glucocorticoids, which are widely used in ALL therapy. Thus, CREBBP mutations may impair the response of leukemic cells to glucocorticoids and influence treatment responsiveness, and therapeutic approaches modifying acetylation may reverse glucocorticoid resistance. Mutations in TP53, which are otherwise infrequent in ALL, are also enriched in relapsed ALL (116).

\section{Inherited genetic variation and risk of ALL}

Several candidate gene studies have implicated inherited genetic variation, for example in the folate metabolic pathway, in the risk of developing leukemia (117). Multiple genome-wide association studies have reproducibly identified variations at several genomic loci that are associated with ALL risk, including IKZF1, ARID5B (encoding the AT-rich interactive domain $5 \mathrm{~B}$ transcription factor), and CEBPE (encoding the transcription factor CCAAT/enhancerbinding protein, epsilon) $(118,119)$. All three genes encode proteins considered important for normal lymphoid development and/or lymphoid leukemogenesis. CEBPE is a target of translocations in ALL, and Arid5b knockout mice exhibit defects in the B lymphoid compartment (120). Although the functional effects of these inherited variants are poorly understood, the $I K Z F 1$ variants may influence level of IKZF1 gene expression (119), suggesting a direct role for these variants in disease susceptibility.

\section{Conclusions}

The use of contemporary technologies to identify genetic alterations in B-ALL has been tremendously informative, but clearly much work remains to be done. Microarray and candidate gene sequencing approaches are incapable of identifying mutations at nucleotidelevel resolution, and initial second-generation sequencing of ALL genomes has identified multiple new genes targeted in ALL. An additional high-priority area of investigation is ALL in older children, adolescents, and adults, who typically have a substantially inferior prognosis. Profiling of ALL in these age groups, as well as in additional uncommon but high-risk ALL subtypes, is ongoing and likely to yield additional insights and therapeutic approaches.

\section{Acknowledgments}

The author thanks members of his laboratory and colleagues at St. Jude Children's Research Hospital, the Children's Oncology Group, and the National Cancer Institute Therapeutically Applicable Research to Generate Effective Treatments (TARGET) Initiative, who have contributed to the studies described in this Review, and apologizes to the many investigators whose important studies could not be cited due to space restrictions. The author is supported by the American Lebanese Syrian Associated Charities of St. Jude Children's Research Hospital and by funding from the National Cancer Institute, the American Society of Hematology, the American Association of Cancer Research, and Stand Up to Cancer. The author is a Pew Scholar in the Biomedical Sciences and a St. Baldrick's Scholar.

Address correspondence to: Charles G. Mullighan, Department of Pathology, St. Jude Children's Research Hospital, 262 Danny Thomas Place, Mail Stop 342, Room 4047G, Memphis, Tennessee 38105, USA. Phone: 901.595.3387; Fax: 901.595.5947; E-mail: charles.mullighan@stjude.org.
1. Pui CH, Robison LL, Look AT. Acute lymphoblastic leukaemia. Lancet. 2008;371(9617):1030-1043.

2. Harrison CJ. Cytogenetics of paediatric and adolescent acute lymphoblastic leukaemia. Br J Haematol. 2009;144(2):147-156.

3. Harrison CJ, Foroni L. Cytogenetics and molecular genetics of acute lymphoblastic leukemia. Rev Clin Exp Hematol. 2002;6(2):91-113.

4. Nachman JB, et al. Outcome of treatment in children with hypodiploid acute lymphoblastic leukemia. Blood. 2007;110(4):1112-1115.

5. Shurtleff SA, et al. TEL/AML1 fusion resulting from a cryptic $t(12 ; 21)$ is the most common genetic lesion in pediatric ALL and defines a subgroup of patients with an excellent prognosis. Leukemia. 1995;9(12):1985-1989.

6. Bohlander SK. ETV6: a versatile player in leukemogenesis. Semin Cancer Biol. 2005;15(3):162-174.

7. Okuda T, van Deursen J, Hiebert SW, Grosveld G, Downing JR. AML1, the target of multiple chromosomal translocations in human leukemia, is essential for normal fetal liver hematopoiesis. Cell. 1996;84(2):321-330.

8. Song WJ, et al. Haploinsufficiency of CBFA2 causes familial thrombocytopenia with propensity to develop acute myelogenous leukaemia. Nat Genet. 1999;23(2):166-175.

9. Roumier C, Fenaux P, Lafage M, Imbert M, Eclache V, Preudhomme C. New mechanisms of AML1 gene alteration in hematological malignancies. Leukemia. 2003;17(1):9-16.

10. Zhang J, et al. The genetic basis of early T-cell precursor acute lymphoblastic leukaemia. Nature. 2012;481(7380):157-163.

11. Wang LC, Kuo F, Fujiwara Y, Gilliland DG, Golub TR, Orkin SH. Yolk sac angiogenic defect and intraembryonic apoptosis in mice lacking the Ets-related factor TEL. EMBO J. 1997;16(14):4374-4383.
12. Hiebert SW, et al. The $\mathrm{t}(12 ; 21)$ translocation converts AML-1B from an activator to a repressor of transcription. Mol Cell Biol. 1996;16(4):1349-1355.

13. Torrano V, Procter J, Cardus P, Greaves M, Ford AM. ETV6-RUNX1 promotes survival of early B lineage progenitor cells via a dysregulated erythropoietin receptor. Blood. 2011;118(18):4910-4918.

14. Andreasson P, Schwaller J, Anastasiadou E, Aster J, Gilliland DG. The expression of ETV6/CBFA2 (TEL/AML1) is not sufficient for the transformation of hematopoietic cell lines in vitro or the induction of hematologic disease in vivo. Cancer Genet Cytogenet. 2001;130(2):93-104.

15. Morrow M, Horton S, Kioussis D, Brady HJ, Williams O. TEL-AML1 promotes development of specific hematopoietic lineages consistent with preleukemic activity. Blood. 2004;103(10):3890-3896.

16. Wiemels JL, et al. Prenatal origin of acute lymphoblastic leukaemia in children. Lancet. 1999; 354(9189):1499-1503.

17. Mullighan CG, et al. Genome-wide analysis of genetic alterations in acute lymphoblastic leukaemia. Nature. 2007;446(7137):758-764.

18. Parker $\mathrm{H}$, et al. The complex genomic profile of ETV6-RUNX1 positive acute lymphoblastic leukemia highlights a recurrent deletion of TBL1XR1. Genes Chromosomes Cancer. 2008;47(12):1118-1125.

19. Kuiper RP, et al. High-resolution genomic profiling of childhood ALL reveals novel recurrent genetic lesions affecting pathways involved in lymphocyte differentiation and cell cycle progression. Leukemia. 2007;21(6):1258-1266.

20. Bateman CM, et al. Acquisition of genome-wide copy number alterations in monozygotic twins with acute lymphoblastic leukemia. Blood. 2010; 115(17):3553-3558.

21. Hunger SP. Chromosomal translocations involving the E2A gene in acute lymphoblastic leukemia: clinical features and molecular pathogenesis. Blood. 1996;87(4):1211-1224.

22. Zhuang Y, Soriano P, Weintraub H. The helix-loophelix gene E2A is required for $\mathrm{B}$ cell formation. Cell. 1994;79(5):875-884.

23. Bain $G$, et al. E2A deficiency leads to abnormalities in alphabeta T-cell development and to rapid development of T-cell lymphomas. Mol Cell Biol. 1997; 17(8):4782-4791.

24. Sanyal M, et al. B-cell development fails in the absence of the Pbx1 proto-oncogene. Blood. 2007; 109(10):4191-4199.

25. Lu Q, Kamps MP. Heterodimerization of Hox proteins with $\mathrm{Pbx} 1$ and oncoprotein $\mathrm{E} 2 \mathrm{a}-\mathrm{Pbx} 1$ generates unique DNA-binding specifities at nucleotides predicted to contact the $\mathrm{N}$-terminal arm of the Hox homeodomain--demonstration of Hoxdependent targeting of E2a-Pbx1 in vivo. Oncogene. 1997;14(1):75-83.

26. Inaba $\mathrm{T}$, et al. Fusion of the leucine zipper gene HLF to the E2A gene in human acute B-lineage leukemia. Science. 1992;257(5069):531-534.

27. de Boer J, et al. The E2A-HLF oncogenic fusion protein acts through $\mathrm{Lmo} 2$ and $\mathrm{Bcl}-2$ to immortalize hematopoietic progenitors. Lenkemia. 2011; 25(2):321-330.

28. Heltemes-Harris LM, et al. Ebf1 or Pax5 haploinsufficiency synergizes with STAT5 activation to initiate acute lymphoblastic leukemia. J Exp Med. 2011;208(6):1135-1149.

29. Ribeiro RC, Abromowitch M, Raimondi SC, Murphy SB, Behm F, Williams DL. Clinical and biologic hallmarks of the Philadelphia chromosome in childhood acute lymphoblastic leukemia. Blood. 1987;70(4):948-953.

30. Melo JV. The diversity of BCR-ABL fusion proteins and their relationship to leukemia phenotype. Blood. 1996;88(7):2375-2384. 
31. Van Etten RA. Studying the pathogenesis of BCR-ABL+ leukemia in mice. Oncogene. 2002 . 21(56):8643-8651.

32. Duy C, et al. BCL6 enables $\mathrm{Ph}+$ acute lymphoblastic leukaemia cells to survive BCR-ABL1 kinase inhibition. Nature. 2011;473(7347):384-388.

33. Mullighan CG, et al. BCR-ABL1 lymphoblastic leukaemia is characterized by the deletion of Ikaros. Nature. 2008;453(7191):110-114.

34. Iacobucci I, et al. Identification and molecular characterization of recurrent genomic deletions on $7 \mathrm{p} 12$ in the IKZF1 gene in a large cohort of BCR-ABL1positive acute lymphoblastic leukemia patients: on behalf of Gruppo Italiano Malattie Ematologiche dell'Adulto Acute Leukemia Working Party (GIMEMA AL WP). Blood. 2009;114(10):2159-2167.

35. Georgopoulos $\mathrm{K}$, et al. The Ikaros gene is required for the development of all lymphoid lineages. Cell. 1994;79(1):143-156.

36. Molnar A, Georgopoulos K. The Ikaros gene encodes a family of functionally diverse zinc finger DNA-binding proteins. Mol Cell Biol. 1994;14(12):8292-8303.

37. Virely C, et al. Haploinsufficiency of the IKZF1 (IKAROS) tumor suppressor gene cooperates with BCR-ABL in a transgenic model of acute lymphoblastic leukemia. Leukemia. 2010;24(6):1200-1204.

38. Crist W, et al. Philadelphia chromosome positive childhood acute lymphoblastic leukemia: clinical and cytogenetic characteristics and treatment outcome. A Pediatric Oncology Group study. Blood. 1990;76(3):489-494.

39. Arico M, et al. Clinical outcome of children with newly diagnosed Philadelphia chromosome-positive acute lymphoblastic leukemia treated between 1995 and 2005. J Clin Oncol. 2010;28(31):4755-4761.

40. Druker BJ, et al. Activity of a specific inhibitor of the BCR-ABL tyrosine kinase in the blast crisis of chronic myeloid leukemia and acute lymphoblastic leukemia with the Philadelphia chromosome. N Engl J Med. 2001;344(14):1038-1042.

41. Druker BJ, et al. Efficacy and safety of a specific inhibitor of the BCR-ABL tyrosine kinase in chronic myeloid leukemia. N Engl J Med. 2001; 344(14):1031-1037.

42. Schultz KR, et al. Improved early event-free survival with imatinib in Philadelphia chromosomepositive acute lymphoblastic leukemia: a children's oncology group study. J Clin Oncol. 2009; 27(31):5175-5181.

43. Pfeifer $\mathrm{H}$, et al. Prevalence and dynamics of bcr-abl kinase domain mutations during imatinib treatment differ in patients with newly diagnosed and recurrent bcr-abl positive acute lymphoblastic leukemia. Leukemia. 2012;26(7):1475-1481.

44. Chen CS, et al. Molecular rearrangements on chromosome $11 \mathrm{q} 23$ predominate in infant acute lymphoblastic leukemia and are associated with specific biologic variables and poor outcome. Blood. 1993; 81(9):2386-2393

45. Pui CH, et al. 11q23/MLL rearrangement confers a poor prognosis in infants with acute lymphoblastic leukemia. J Clin Oncol. 1994;12(5):909-915.

46. Pui CH, Kane JR, Crist WM. Biology and treatment of infant leukemias. Leukemia. 1995;9(5):762-769.

47. Pui CH, et al. Secondary acute myeloid leukemia in children treated for acute lymphoid leukemia. NEngl J Med. 1989;321(3):136-142.

48. Chessels JM, Swansbury GJ, Reeves B, Bailey CC, Richards SM. Cytogenetics and prognosis in childhood lymphoblastic leukaemia: results of MRC UKALL X. Medical Research Council Working Party in Childhood Leukaemia. BrJ Haematol. 1997; 99(1):93-100.

49. Moorman AV, et al. Karyotype is an independent prognostic factor in adult acute lymphoblastic leukemia (ALL): analysis of cytogenetic data from patients treated on the Medical Research Council (MRC) UKALLXII/Eastern Cooperative
Oncology Group (ECOG) 2993 trial. Blood. 2007; 109(8):3189-3197.

50. Meyer C, et al. The MLL recombinome of acute leukemias. Lenkemia. 2006;20(5):777-784.

51. Chessells JM, et al. Clinical features, cytogenetics and outcome in acute lymphoblastic and myeloid leukaemia of infancy: report from the MRC Childhood Leukaemia working party. Lenkemia. 2002; 16(5):776-784.

52. Moorman AV, et al. No prognostic effect of additional chromosomal abnormalities in children with acute lymphoblastic leukemia and $11 \mathrm{q} 23$ abnormalities. Leukemia. 2005;19(4):557-563.

53. Ziemin-van der Poel S, McCabe NR, Gill HJ, Espinosa R. Identification of a gene, MLL, that spans the breakpoint in $11 \mathrm{q} 23$ translocations associated with human leukemias. Proc Natl Acad Sci U S A. 1991;88(23):10735-10739.

54. Milne TA, et al. MLL targets SET domain methyltransferase activity to Hox gene promoters. Mol Cell. 2002;10(5):1107-1117.

55. Bardini M, et al. DNA copy-number abnormalities do not occur in infant ALL with $\mathrm{t}(4 ; 11) / \mathrm{MLL}-\mathrm{AF} 4$. Leukemia. 2010;24(1):169-176.

56. Andersson AK, et al. Whole genome sequence analysis of 22 MLL rearranged infant acute lymphoblastic leukemias reveals remarkably few somatic mutations: a report from the St. Jude Children's Research Hospital - Washington University Pediatric Cancer Genome Project. ASH Annual Meeting Abstracts. 2011;118:69.

57. Armstrong SA, et al. MLL translocations specify a distinct gene expression profile that distinguishes a unique leukemia. Nat Genet. 2002;30(1):41-47.

58. Faber J, et al. HOXA9 is required for survival in human MLL-rearranged acute leukemias. Blood. 2009;113(11):2375-2385

59. Armstrong SA, et al. Inhibition of FLT3 in MLL. Validation of a therapeutic target identified by gene expression based classification. Cancer Cell. 2003; 3(2):173-183

60. Stubbs MC, et al. MLL-AF9 and FLT3 cooperation in acute myelogenous leukemia: development of a model for rapid therapeutic assessment. Leukemia. 2008;22(1):66-77.

61. Stumpel DJ, et al. Specific promoter methylation identifies different subgroups of MLL-rearranged infant acute lymphoblastic leukemia, influences clinical outcome, and provides therapeutic options. Blood. 2009;114(27):5490-5498.

62. Stumpel DJ, et al. Hypermethylation of specific microRNA genes in MLL-rearranged infant acute lymphoblastic leukemia: major matters at a micro scale. Leukemia. 2011;25(3):429-439.

63. Krivtsov AV, et al. H3K79 methylation profiles define murine and human MLL-AF4 leukemias. Cancer Cell. 2008;14(5):355-368.

64. Yan L, et al. Clinical, immunophenotypic, cytogenetic, and molecular genetic features in 117 adult patients with mixed-phenotype acute leukemia defined by WHO-2008 classification [published online ahead of print May 11, 2012]. Haematologica. doi:10.3324/haematol.2012.064485.

65. Jo SY, Granowicz EM, Maillard I, Thomas D, Hess JL. Requirement for Dot1l in murine postnatal hematopoiesis and leukemogenesis by MLL translocation. Blood. 2011;117(18):4759-4768.

66. Daigle SR, et al. Selective killing of mixed lineage leukemia cells by a potent small-molecule DOT1L inhibitor. Cancer Cell. 2011;20(1):53-65.

67. Schafer E, et al. Promoter hypermethylation in MLL-r infant acute lymphoblastic leukemia: biology and therapeutic targeting. Blood. 2010;115(23):4798-4809.

68. Milani L, et al. DNA methylation for subtype classification and prediction of treatment outcome in patients with childhood acute lymphoblastic leukemia. Blood. 2010;115(6):1214-1225.

69. Dyer MJ, et al. Immunoglobulin heavy chain (IGH) locus chromosomal translocations in B-cell precursor acute lymphoblastic leukemia (BCP-ALL): rare clinical curios or potent genetic drivers? Blood. 2010;115(8):1490-1499.

70. Klapproth K, Wirth T. Advances in the understanding of MYC-induced lymphomagenesis. Br J Haematol. 2010;149(4):484-497.

71. Russell LJ, et al. Deregulated expression of cytokine receptor gene, CRLF2, is involved in lymphoid transformation in B-cell precursor acute lymphoblastic leukemia. Blood. 2009;114(13):2688-2698.

72. Mullighan CG, et al. Rearrangement of CRLF2 in B-progenitor- and Down syndrome-associated acute lymphoblastic leukemia. Nat Genet. 2009; 41(11):1243-1246.

73. Yoda A, et al. Functional screening identifies CRLF2 in precursor B-cell acute lymphoblastic leukemia. Proc Natl Acad Sci U S A. 2010;107(1):252-257.

74. Harvey RC, et al. Rearrangement of CRLF2 is associated with mutation of JAK kinases, alteration of IKZF1, Hispanic/Latino ethnicity, and a poor outcome in pediatric B-progenitor acute lymphoblastic leukemia. Blood. 2010;115(26):5312-5321.

75. Hertzberg L, et al. Down syndrome acute lymphoblastic leukemia: a highly heterogeneous disease in which aberrant expression of CRLF2 is associated with mutated JAK2: a report from the iBFM Study Group. Blood. 2010;115(5):1006-1017.

76. Bercovich D, et al. Mutations of JAK2 in acute lymphoblastic leukaemias associated with Down's syndrome. Lancet. 2008;372(9648):1484-1492.

77. Mullighan CG, et al. JAK mutations in high-risk childhood acute lymphoblastic leukemia. Proc Natl Acad Sci U S A. 2009;106(23):9414-9418.

78. Cario G, et al. Presence of the P2RY8-CRLF2 rearrangement is associated with a poor prognosis in non-high-risk precursor B-cell acute lymphoblastic leukemia in children treated according to the ALLBFM 2000 protocol. Blood. 2010;115(26):5393-5397.

79. Shochat C, et al. Gain-of-function mutations in interleukin-7 receptor-alpha (IL7R) in childhood acute lymphoblastic leukemias. J Exp Med. 2011; 208(5):901-908

80. Zenatti PP, et al. Oncogenic IL7R gain-of-function mutations in childhood T-cell acute lymphoblastic leukemia. Nat Genet. 2011;43(10):932-939.

81. Mullighan CG, et al. Deletion of IKZF1 and prognosis in acute lymphoblastic leukemia. $N$ Engl J Med. 2009;360(5):470-480.

82. Den Boer ML, et al. A subtype of childhood acute lymphoblastic leukaemia with poor treatment outcome: a genome-wide classification study. Lancet Oncol. 2009;10(2):125-134.

83. Loh ML, et al. A BCR-ABL1-like gene expression profile confers a poor prognosis in patients with high-risk acute lymphoblastic leukemia (HRALL): a report from Children's Oncology Group (COG) AALL0232. ASH Annual Meeting Abstracts. 2011;118:743

84. Roberts KG, et al. Genetic alterations activating kinase and cytokine receptor signaling in high rish acute lymphoblastic leukemia. Cancer Cell. 2012; 22(2):153-166.

85. Harewood L, et al. Amplification of AML1 on a duplicated chromosome 21 in acute lymphoblastic leukemia: a study of 20 cases. Lenkemia. 2003; 17(3):547-553.

86. Robinson HM, Harrison CJ, Moorman AV, Chudoba I, Strefford JC. Intrachromosomal amplification of chromosome 21 (iAMP21) may arise from a breakage-fusion-bridge cycle. Genes Chromosomes Cancer. 2007;46(4):318-326.

87. Inukai $\mathrm{T}$, et al. Clinical significance of early $\mathrm{T}$-cell precursor acute lymphoblastic leukaemia: results of the Tokyo Children's Cancer Study Group Study L99-15. Br J Haematol. 2012;156(3):358-365.

88. Attarbaschi A, et al. Minimal residual disease values discriminate between low and high relapse risk 
in children with B-cell precursor acute lymphoblastic leukemia and an intrachromosomal amplification of chromosome 21: the Austrian and German acute lymphoblastic leukemia Berlin-FrankfurtMunster (ALL-BFM) trials. J Clin Oncol. 2008; 26(18):3046-3050.

89. Harrison CJ, et al. Three distinct subgroups of hypodiploidy in acute lymphoblastic leukaemia. Br J Haematol. 2004;125(5):552-559.

90. Holmfeldt L, et al. Genome-wide analysis of genetic alterations in hypodiploid acute lymphoblastic leukemia identifies a high frequency of mutations targeting the IKAROS gene family and Ras signaling. ASH Annual Meeting Abstracts. 2010;116:411.

91. Mullighan CG. Single nucleotide polymorphism microarray analysis of genetic alterations in cancer. Methods Mol Biol. 2011;730:235-258.

92. Yeoh EJ, et al. Classification, subtype discovery, and prediction of outcome in pediatric acute lymphoblastic leukemia by gene expression profiling. Cancer Cell. 2002;1(2):133-143.

93. Bousquet M, et al. A novel PAX5-ELN fusion protein identified in B-cell acute lymphoblastic leukemia acts as a dominant negative on wild-type PAX5. Blood. 2007;109(8):3417-3423.

94. Nebral K, et al. Incidence and diversity of PAX5 fusion genes in childhood acute lymphoblastic leukemia. Leukemia. 2009;23(1):134-143.

95. Iacobucci I, et al. The PAX5 gene is frequently rearranged in BCR-ABL1-positive acute lymphoblastic leukemia but is not associated with outcome. A report on behalf of the GIMEMA Acute Leukemia Working Party. Haematologica. 2010;95(10):1683-1690.

96. Kuiper RP, et al. IKZF1 deletions predict relapse in uniformly treated pediatric precursor B-ALL. Lenkemia. 2010;24(7):1258-1264.

97. Kawamata N, et al. Molecular allelokaryotyping of pediatric acute lymphoblastic leukemias by high-resolution single nucleotide polymorphism oligonucleotide genomic microarray. Blood. 2008; 111(2):776-784.

98. Yamamoto T, et al. PTPN11, RAS and FLT3 mutations in childhood acute lymphoblastic leukemia. Leuk Res. 2006;30(9):1085-1089.

99. Paulsson K, et al. Mutations of FLT3, NRAS, KRAS, and PTPN11 are frequent and possibly mutually exclusive in high hyperdiploid childhood acute lymphoblastic leukemia. Genes Chromosomes Cancer. 2008;47(1):26-33.
100.Zhang J, et al. Key pathways are frequently mutated in high-risk childhood acute lymphoblastic leukemia: a report from the Children's Oncology Group. Blood. 2011;118(11):3080-3087.

101. Raimondi SC, Pui CH, Head DR, Rivera GK, Behm FG. Cytogenetically different leukemic clones at relapse of childhood acute lymphoblastic leukemia. Blood. 1993;82(2):576-580.

102. Maloney KW, McGavran L, Odom LF, Hunger SP. Acquisition of p16(INK4A) and p15(INK4B) gene abnormalities between initial diagnosis and relapse in children with acute lymphoblastic leukemia. Blood. 1999;93(7):2380-2385.

103. Irving JA, Minto L, Bailey S, Hall AG. Loss of heterozygosity and somatic mutations of the glucocorticoid receptor gene are rarely found at relapse in pediatric acute lymphoblastic leukemia but may occur in a subpopulation early in the disease course. Cancer Res. 2005;65(21):9712-9718.

104.Mullighan CG, et al. Genomic analysis of the clonal origins of relapsed acute lymphoblastic leukemia. Science. 2008;322(5906):1377-1380.

105. Yang JJ, et al. Genome-wide copy number profiling reveals molecular evolution from diagnosis to relapse in childhood acute lymphoblastic leukemia. Blood. 2008;112(10):4178-4183.

106.van Delft FW, et al. Clonal origins of relapse in ETV6-RUNX1 acute lymphoblastic leukemia. Blood. 2011;117(23):6247-6254.

107. Kawamata N, et al. Molecular allelokaryotyping of relapsed pediatric acute lymphoblastic leukemia. Int J Oncol. 2009;34(6):1603-1612.

108. Kuster L, et al. ETV6/RUNX1-positive relapses evolve from an ancestral clone and frequently acquire deletions of genes implicated in glucocorticoid signaling. Blood. 2011;117(9):2658-2667.

109.Szczepanski T, et al. Late recurrence of childhood T-cell acute lymphoblastic leukemia frequently represents a second leukemia rather than a relapse: first evidence for genetic predisposition. J Clin Oncol. 2011;29(12):1643-1649.

110. Notta F, et al. Evolution of human BCR-ABL1 lymphoblastic leukaemia-initiating cells. Nature. 2011; 469(7330):362-367.

111.Anderson K, et al. Genetic variegation of clonal architecture and propagating cells in leukaemia. Nature. 2011;469(7330):356-361.

112.Mullighan CG, et al. CREBBP mutations in relapsed acute lymphoblastic leukaemia. Nature. 2011;
471(7337):235-239.

113. Goodman RH, Smolik S. CBP/p300 in cell growth, transformation, and development. Genes Dev. 2000;14(13):1553-1577.

114.Inthal A, et al. CREBBP HAT domain mutations prevail in relapse cases of high hyperdiploid childhood acute lymphoblastic leukemia [published online ahead of print March 5, 2012]. Lenkemia. doi:10.1038/leu.2012.60.

115.Pasqualucci L, et al. Inactivating mutations of acetyltransferase genes in B-cell lymphoma. Nature. 2011;471(7337):189-195.

116. Hof J, et al. Mutations and deletions of the TP53 gene predict nonresponse to treatment and poor outcome in first relapse of childhood acute lymphoblastic leukemia. JClin Oncol. 2011;29(23):3185-3193.

117. Koppen IJ, Hermans FJ, Kaspers GJ. Folate related gene polymorphisms and susceptibility to develop childhood acute lymphoblastic leukaemia. BrJ Haematol. 2010;148(1):3-14

118. Trevino LR, et al. Germline genomic variants associated with childhood acute lymphoblastic leukemia. Nat Genet. 2009;41(9):1001-1005.

119.Papaemmanuil E, et al. Loci on 7 p $12.2,10 q 21.2$ and $14 \mathrm{q} 11.2$ are associated with risk of childhood acute lymphoblastic leukemia. Nat Genet. 2009; 41(9):1006-1010.

120.Lahoud MH, et al. Gene targeting of Desrt, a novel ARID class DNA-binding protein, causes growth retardation and abnormal development of reproductive organs. Genome Res. 2001;11(8):1327-1334.

121. Pui CH, et al. Treating childhood acute lymphoblastic leukemia without cranial irradiation. NEngl JMed. 2009;360(26):2730-2741.

122.Pui CH, et al. Results of therapy for acute lymphoblastic leukemia in black and white children. JAMA. 2003;290(15):2001-2007.

123.Jeha $S$, et al. Increased risk for CNS relapse in pre-B cell leukemia with the $\mathrm{t}(1 ; 19) / \mathrm{TCF} 3-\mathrm{PBX} 1$. Leukemia. 2009;23(8):1406-1409.

124.Mullighan CG, et al. ERG deletions define a novel subtype of B-progenitor acute lymphoblastic leukemia. ASH Annual Meeting Abstracts. 2007;110:691.

125.Martinelli G, et al. IKZF1 (Ikaros) deletions in BCRABL1-positive acute lymphoblastic leukemia are associated with short disease-free survival and high rate of cumulative incidence of relapse: a GIMEMA AL WP report. J Clin Oncol. 2009;27(31):5202-5207. 